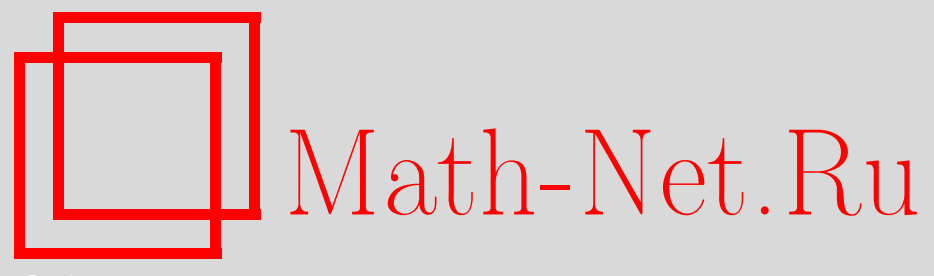

Ал. Б. Замолодчиков, Ю. Ишимото, Массивный майорановский фермион, взаимодействующий с двумерной гравитацией, и модель Изинга на случайной решетке, TMФ, 2006, том 147, номер 3, 372-398

DOI: https://doi.org/10.4213/tmf1985

Использование Общероссийского математического портала Math-Net.Ru подразумевает, что вы прочитали и согласны с пользовательским соглашением http://www . mathnet.ru/rus/agreement

Параметры загрузки:

IP : 34.229 .45 .116

26 апреля 2023 г., 14:06:24

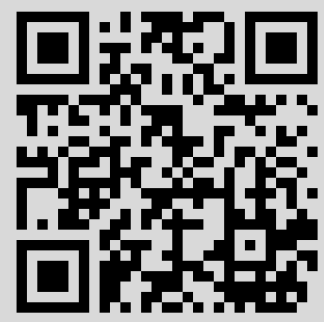




\title{
МАССИВНЫЙ МАЙОРАНОВСКИЙ ФЕРМИОН, ВЗАИМОДЕЙСТВУЮЩИЙ С ДВУМЕРНОЙ ГРАВИТАЦИЕЙ, И МОДЕЛЬ ИЗИНГА НА СЛУЧАЙНОЙ РЕШЕТКЕ
}

\begin{abstract}
Рассматривается статистическая сумма модели двумерного свободного массивного майорановского фермиона, взаимодействующего с квантованной метрикой топологии сферы. Путем добавления произвольной конформной "материи-наблюдателя" можно осуществлять контроль над полным центральным зарядом полей материи. Это порождает интересное непрерывно параметризованное семейство критических точек и допускает квазиклассический предел. Теория поля Лиувилля играет роль эффективной теории квантовой гравитации. Масштабная функция сферы преобразований вычисляется приближенно, но с хорошей численной точностью почти во всем диапазоне изменения параметра “материи-наблюдателя". Впечатляющий результат сравнения с предсказаниями точно решаемой матричной модели приводит к более общей статистической модели на случайной решетке, которая, вероятнее всего, не решается с помощью техники матричных моделей и обладает более общей структурой критического поведения. Численные симуляции или экстраполяции рядов, может быть, позволят обнаружить новое семейство функций скейлинга.
\end{abstract}

Ключевые слова: теория поля Лиувилля, двумерная гравитация, модель Изинга, случайная решетка.

\section{1. ВВЕДЕНИЕ}

Недавние исследования показали, что теория поля Лиувилля (ТПЛ) представляет собой эффективный метод исследования задач двумерной квантовой гравитации [1]. В настоящее время полагают, что полевая теория двумерной гравитации, основанная на ТПЛ, дает эффективное описание универсального критического поведения в определенных моделях статистической физики, называемых спиновыми системами на случайных решетках. Эти системы были сначала введены скорее как

*Laboratoire de Physique Théorique et Astroparticules Université Montpellier II Pl.E. Bataillon, 34095 Montpellier, France; Институт экспериментальной и теоретической физики, Москва, Россия. E-mail: Aliocha.ZAMOLODCHIKOV@lpta.univ-montp2.fr

${ }^{\dagger}$ Kawai Theoretical Laboratory, RIKEN, Saitama, Japan 
модели собственно непрерывной двумерной гравитации [2]-[5], и поэтому мотивация их изучения в рамках статистической механики связана в основном с поляковской формулировкой струнной теории [6]. Модели на случайных решетках (MCP) можно рассматривать как обычные решеточные системы, но заданные на общем графе фиксированной топологии, при этом сам граф представляет собой динамическую степень свободы, по которой производится усреднение в статистической сумме. С этой точки зрения термин "динамическая решетка" или "динамическая триангуляция" [5] представляется более правильным, чем традиционное название МСР. Уже весьма давно было замечено, что такая "наивная" версия исчисления Редже [7] при больших размерах решетки допускает интересное критическое поведение (см., например, [4]). Более того, оказывается, что критические индексы могут быть сведены к индексам, появляющимся в непрерывном описании с помощью теоретико-полевого функционального интеграла по всем метрикам на поверхности [8]. Было обнаружено, что ТПЛ может служить удобным инструментом исследования этой задачи, особенно в формулировке Давида-Дистлера-Каваи [9].

Дальнейшее развитие теоретико-полевых подходов к двумерной гравитации потребовало существенно более глубокого понимания ТПЛ как конформной теории поля (KTП). Несмотря на некоторые попытки, основанные на "аналитическом продолжении по числу интегрирований" [10], достичь значительного прогресса не удалось. Может быть, причиной этого является интуитивная, но весьма распространенная идея о том, что структуры, ранее изученные в рациональных КТП, до некоторой степени должны действовать и при описании общих $\mathrm{KT \Pi}^{1)}$. Наконец, Дорн и Отто в 1992 г. подтвердили существовавшую ранее веру в то, что ТПЛ представляет собой решаемую КТП и нашли явное выражение для трехточечной корреляционной функции [11]. К сожалению, до последнего времени этот предмет был почти полностью заброшен, и дальнейшие исследования шли довольно вяло [12], [13]. Это, возможно, было связано с тем, что приложение новых результатов к струнной теории остается проблематичным, кроме некоторых граничных версий [14].

В данной статье мы развиваем противоположную сторону ТПЛ (и общей непрерывной двумерной гравитации), а именно ее роль как эффективной полевой теории критического поведения МСР. Многие интересные модели на динамических решетках допускают точные решения с использованием методов матричных моделей [15]. Первые приложения к теориям двумерной гравитации появились уже в работе [2]. Это привело к другой популярной идее, заключающейся в том, что в терминах матричных моделей критического поведения можно описать практически все классы универсальности МСР, а следовательно, и все возможные модели непрерывной двумерной гравитации ${ }^{2)}$. Мы, однако, находим эту идею не бесспорной, и одна из основных мотиваций нашего исследования состоит в обосновании наших сомнений. Для этого мы исследуем теорию двумерного массивного майорановского фермиона, взаимодействующего с квантованной гравитацией. Эта теория поля, как

1)Эта идея до сих пор находит последователей, особенно среди молодых теоретиков. Это безусловно связано с недостатками изложения в существующих книгах, посвященных КТП.

2) Довольно часто термины "матричные модели" и “двумерная гравитация" используются как синонимы, особенно в исследованиях, близких к теории струн. 
считается, представляет собой непрерывное описание модели Изинга на случайной решетке (МИСР), впервые сформулированной и решенной Казаковым [16] (более детальное исследование см. в работе [17]). В самом деле, известно, что все критические индексы МИСР [8] воспроизводятся правильным образом в описании ТПЛ. В данной работе мы не только рассматриваем критические индексы, но и пытаемся вычислить функцию скейлинга, связанную со статистической суммой в геометрии сферы в случае массивной (возмущенной) материи. До настоящего времени в подходе ТПЛ получить удовлетворительный результат для таких функций скейлинга не удавалось. Вместо них оказалось возможным построить некоторые критические корреляционные функции (см., например, [10], [18] или [19] по поводу более свежих исследований) и, тем самым, воспроизвести несколько первых членов конформного разложения в теории возмущений. Тем не менее, в работе [1] было показано, что даже эта ограниченная информация оказывается весьма полезной, если принять определенные гипотезы об аналитической структуре функции скейлинга. На самом деле эти предположения (хотя они и не являются универсальными, и известны несколько контрпримеров) представляются вполне естественными и были проверены в целом ряде частных случаев. В рамках этих аналитических предположений информация, полученная в рамках теории возмущений, может быть обработана путем комбинированного аналитически-численного анализа [1], эффективность которого наблюдалась ранее в случае классической гравитации [20]. Результаты, хотя и оказываются приближенными, зачастую воспроизводят численное описание функции скейлинга со впечатляющей точностью.

В данной работе мы реализуем эту же программу для майорановского фермиона в КТП, возмущенного массивным членом. Сферическую функцию скейлинга, полученную таким образом, можно сравнивать с точной функцией, вычисленной в рамках подхода матричных моделей. На самом деле, мы рассматриваем более общую модель, в которой вводится дополнительная конформная материя, всегда остающаяся в критическом состоянии, но влияющая на величину параметра ТПЛ, который в этом случае можно изменять непрерывно. Мы предлагаем интерпретировать соответствующие функции скейлинга как своего рода обобщение стандартной МИСР, получаемой в результате "наивного" одевания статистических весов "детерминантным" множителем. Такая обобщенная МИСР вряд ли окажется точно решаемой в общем случае, но вполне возможно допускает численные исследования с помощью экстраполяций данных, полученных при конечном $N$ [21], или с использованием расчетов по методу Монте-Карло [5], [22]. Если наше предположение справедливо, могут быть обнаружены новые типы критического поведения, которые никогда ранее не наблюдались в исследованиях на основе матричных моделей.

\section{2. МАССИВНЫЙ ФЕРМИОН, ВЗАИМОДЕЙСТВУЮЩИЙ С ПОЛЕМ ЛИУВИЛЛЯ}

Наша модель задается следующим локальным лагранжианом:

$$
\mathcal{L}_{m}=\mathcal{L}_{\mathrm{L}}(b)+\mathcal{L}_{\text {Ising }}+\mathcal{L}_{\mathrm{sp}}+\frac{m}{2 \pi} \varepsilon e^{2 a \phi},
$$


где $\mathcal{L}_{\mathrm{L}}-$ стандартное действие Лиувилля с космологической постоянной связи $\mu$ и параметром $b$,

$$
\mathcal{L}_{\mathrm{L}}(b)=\frac{1}{4 \pi}\left(\partial_{a} \phi\right)^{2}+\mu e^{2 b \phi} .
$$

Во всем, что имеет отношение к ТПЛ, мы следуем обозначениям работы [12], в частности, мы обозначаем через $V_{a}$ квантовую версию лиувиллевской экспоненты $e^{2 a \phi}$, которая представляет собой лиувиллевское первичное поле размерности $\Delta_{a}^{(\mathrm{L})}=$ $a(Q-a)$. Лиувиллевский центральный заряд (как обычно, $\left.Q=b^{-1}+b\right)$ имеет вид:

$$
c_{\mathrm{L}}=1+6 Q^{2} \text {. }
$$

Тогда $\mathcal{L}_{\text {Ising }}$ представляет собой стандартную теорию для свободного безмассового майорановского фермиона с $c=1 / 2$ :

$$
\mathcal{L}_{\text {Ising }}=\frac{1}{2 \pi}(\psi \bar{\partial} \psi+\bar{\psi} \partial \bar{\psi}) .
$$

Эта нормировка отвечает следующим пропагаторам безмассовых свободных фермионов:

$$
\langle\psi(z) \psi(0)\rangle=\frac{1}{z}, \quad\langle\bar{\psi}(\bar{z}) \bar{\psi}(0)\rangle=\frac{1}{\bar{z}} .
$$

Оператор плотности энергии $\varepsilon=i \bar{\psi} \psi$, играющий роль возмущения в лагранжиане $(2.1)$, имеет размерность $(1 / 2,1 / 2)$. Он нормирован в КТП стандартным обра3ом:

$$
\langle\varepsilon(x) \varepsilon(0)\rangle=\frac{1}{x \bar{x}} .
$$

Наконец, как и в работе [1], добавим "материю-наблюдателя", т.е. КТП с центральным зарядом $c_{\mathrm{sp}}$, чтобы иметь возможность контролировать параметры лиувиллевской гравитации. Эта материя не взаимодействует с членом возмущения, а потому остается конформной, и в нашем формализме она полностью отщепляется от других степеней свободы ${ }^{3)}$. Присутствие этого поля достигается добавлением члена $\mathcal{L}_{\mathrm{sp}}$ в полный лагранжиан. Получающееся при этом семейство теорий двумерной гравитации мы назовем гравитационной моделью Изинга (ГМИ).

В квазиклассическом анализе член взаимодействия в лагранжиане лучше всего записывается в виде

$$
\mathcal{L}_{\text {int }}=\frac{m}{2 \pi}\left(\varepsilon e^{2 a \phi}+\frac{m}{16 a^{2}} e^{4 a \phi}\right) .
$$

Второе слагаемое в скобках представляет собой "индуцированный" контрчлен, который необходим для придания определенной размерности возмущающему полю.

Как обычно, выполняются уравнения равновесия

$$
c_{\mathrm{sp}}+\frac{3}{2}+6 Q^{2}=26, \quad a(Q-a)=\frac{1}{2} .
$$

Первое уравнение легко преобразуется к виду

$$
6 Q^{2}=\frac{49}{2}-c_{\mathrm{sp}}
$$

\footnotetext{
3) Напомним, что такое отщепление специфично для случая сферической топологии. В менее тривиальных топологиях остается остаточное взаимодействие через модули конформных структур.
} 
Это соотношение определяет параметр $b$ нашей модели в терминах центрального заряда фоновой материи. В частности, квазиклассический режим $b \rightarrow 0$ достигается при $c_{\mathrm{sp}} \rightarrow-\infty$, а случай “чистой модели Изинга" $c_{\mathrm{sp}}=0$ отвечает $b^{2}=3 / 4$.

В соответствии со здравым смыслом (почерпнутым из опыта работы в матричных моделях, а также из квазиклассических соображений) во втором из уравнений (2.8) следует выбирать наименьшее из двух решений

$$
a=\frac{Q}{2}-\sqrt{\frac{Q^{2}-2}{4}} .
$$

Для чистой модели Изинга имеем

$$
a=\frac{b}{3},
$$

в то время как в квазиклассическом режиме

$$
a=\frac{b}{2}-\frac{b^{3}}{4}+O\left(b^{7}\right)
$$

Из самосогласованности ТПЛ следует, что параметр $b$ должен оставаться вещественным. Это ограничивает область значений параметра $c_{\mathrm{sp}}$ : $-\infty<c_{\mathrm{sp}} \leqslant 1 / 2$. Внутри этого интервала мы выбираем в качестве $b$ наименьшее решение первого уравнения (2.8), так что всегда имеем $0<b^{2} \leqslant 1$.

\section{3. СТАТИСТИЧЕСКАЯ СУММА}

Масштабное поведение статистической суммы $Z(m, \mu)$ в топологии сферы (род ноль) имеет вид

$$
Z(m, \mu)=\mu^{Q / b} G_{\text {sphere }}\left(\frac{m}{\mu^{s}}\right),
$$

где функция скейлинга $G_{\text {sphere }}$ зависит от масштабно-инвариантной комбинации констант связи. Показатель $s$ можно легко найти из масштабной зависимости отдельных членов в (2.1):

$$
s=a b^{-1} .
$$

Этот показатель изменяется от $s=1 / 2-b^{2} / 4+\cdots$ в квазиклассическом пределе $b \rightarrow 0$ до $s=1-\sqrt{1 / 2}=0.29289 \ldots$ при $b^{2}=1$. В чистой модели Изинга $s=1 / 3$.

Формальное разложение в ряд теории возмущений по $\mathcal{L}_{\text {int }}$ дает

$$
Z(m, \mu)=\sum_{n=0}^{\infty} \frac{(-m)^{n}}{(2 \pi)^{n} n !}\left\langle\varepsilon^{n}\right\rangle_{\text {ГМи }}
$$

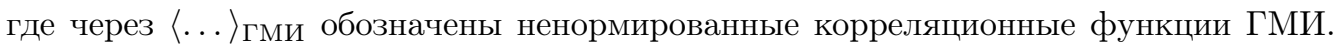
В лиувиллевской гравитации такие $n$-точечные функции задаются интегралами по модулям сферы с $n-3$ выколотыми точками:

$$
\begin{aligned}
\left\langle\varepsilon^{n}\right\rangle_{\text {Гми }}=\int & \left\langle C \bar{C}\left(x_{1}\right) C \bar{C}\left(x_{2}\right) C \bar{C}\left(x_{3}\right)\right\rangle\left\langle\varepsilon\left(x_{1}\right) \ldots \varepsilon\left(x_{n}\right)\right\rangle_{\mathrm{ff}} \times \\
& \times\left\langle V_{a}\left(x_{1}\right) \ldots V_{a}\left(x_{n}\right)\right\rangle_{\mathrm{L}} d^{2} x_{3} \ldots d^{2} x_{n},
\end{aligned}
$$


где $\langle\ldots\rangle_{\mathrm{ff}}$ и $\langle\ldots\rangle_{\mathrm{L}}-$ корреляционные функции в теориях свободного майорановского фермиона и в ТПЛ, соответственно, а $C$ и $\bar{C}$ суть известные из двумерной гравитации и из струнной теории духи, фиксирующие калибровку. Выражение (3.4) не требует дополнительных комментариев для читателя, знакомого со струнной теорией, а неспециалисты могут поискать детали и объяснения в работе [1]. Там также указано, как действовать в случае $n<3$, для которого не пригодно уравнение (3.4) и который поэтому требует специального рассмотрения.

Простой размерный анализ, как обычно, приводит к выводу, что $\left\langle\varepsilon^{n}\right\rangle_{\Gamma м и ~} \sim$ $\mu^{(Q-n a) b}$, и выражение $(3.3)$ представляет собой не что иное, как степенной ряд

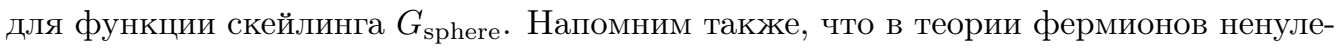
выми будут только корреляционные функции от $\varepsilon$ с четным числом вставок $n=2 k$. Разложение (3.3) можно переписать в виде

$$
\frac{Z(m, \mu)}{Z(0, \mu)}=\sum_{k=0}^{\infty} \frac{a_{2 k}}{(2 \pi)^{2 k}(2 k) !}\left(\frac{m^{2}}{\mu^{2 s}}\right)^{k}
$$

при

$$
a_{n}=Z_{\mathrm{L}}^{-1} \mu^{n s}\left\langle\varepsilon^{n}\right\rangle_{\text {Гми }}
$$

Здесь через $Z_{\mathrm{L}}$ обозначена лиувиллевская статистическая сумма на сфере: $Z_{\mathrm{L}}=\langle I\rangle_{\mathrm{L}}$ (мы предполагаем, что фермионная корреляционная функция нормирована стандартным образом: $\left.\langle I\rangle_{\mathrm{ff}}=1\right)$.

Как и в работе [1], оказывается удобным ввести статистическую сумму $Z_{A}(m)$ с фиксированной площадью,

$$
Z_{A}(m)=A \int_{\uparrow} Z(m, \mu) e^{\mu A} \frac{d \mu}{2 \pi i},
$$

с контуром интегрирования $\uparrow$, проходящим вдоль мнимой оси в комплексной плоскости параметра $\mu$ справа от всех сингулярностей подынтегрального выражения. Эта статистическая сумма допускает разложение

$$
Z_{A}(m)=Z_{A}(0) \sum_{k=0}^{\infty} z_{2 k} \eta^{2 k},
$$

коэффициенты $z_{n}$ которого легко соотнести с коэффициентами в выражении (3.5):

$$
z_{n}=\frac{\pi^{n s} a_{n} \Gamma\left(-b^{-2}-1\right)}{(2 \pi)^{n} n ! \Gamma\left(-b^{-2}-1+n s\right)},
$$

а масштабный параметр $\eta$ имеет вид

$$
\eta=m\left(\frac{A}{\pi}\right)^{s} .
$$

Вследствие наличия дополнительной Г-функции в знаменателе выражения (3.9) ряд (3.8) сходится значительно лучше, чем ряд (3.5). На самом деле, ряд (3.8) сходится абсолютно и задает целую функцию скейлинга от $t=\eta^{2}$ вида

$$
z(t)=\sum_{k=0}^{\infty} z_{2 k} t^{k} .
$$




\section{4. ЧЛЕНЫ РЯДА ТЕОРИИ ВОЗМУЩЕНИЙ}

Как обычно (см., например, [1], [11]), лиувиллевская статистическая сумма может быть восстановлена на основании трехточечной функции в ТПЛ [11], [12]:

$$
Z_{\mathrm{L}}=\left[\pi \mu \gamma\left(b^{2}\right)\right]^{Q / b} \frac{1-b^{2}}{\pi^{3} Q \gamma\left(b^{2}\right) \gamma\left(b^{-2}\right)} .
$$

Аналогично нормировка (2.6) отвечает следующей (ненормированной) двухточечной функции [1]:

$$
\langle\varepsilon \varepsilon\rangle_{\text {гми }}=-\left(\pi \mu \gamma\left(b^{2}\right)\right)^{(Q-2 a) / b} \frac{\gamma\left(2 a b-b^{2}\right) \gamma\left(2 a b^{-1}-b^{-2}\right)}{\pi(Q-2 a)} .
$$

Для первых двух коэффициентов в сумме (3.11) имеем

$$
z_{0}=1, \quad z_{2}=\frac{\gamma\left(b^{2}(2 s-1)\right) \Gamma\left(b^{-2}-1\right) \gamma^{1-2 s}\left(b^{2}\right)}{8 \Gamma\left(1+b^{-2}-2 s\right)} .
$$

Получение следующего коэффициента разложения $z_{4}$ требует более сложных вычислений. По существу он сводится к следующему интегральному представлению:

$$
z_{4}=\frac{1}{24(2 \pi)^{4}} \int\langle\varepsilon(x) \varepsilon(0) \varepsilon(1) \varepsilon(\infty)\rangle_{\mathrm{ff}}\left\langle\left\langle V_{a}(x) V_{a}(0) V_{a}(1) V_{a}(\infty)\right\rangle\right\rangle_{\mathrm{L}}^{(A)} d^{2} x,
$$

где нормированные лиувиллевские корреляционные функции при фиксированной площади имеют вид

$$
\langle\langle\ldots\rangle\rangle_{\mathrm{L}}^{(A)}=\frac{\langle\ldots\rangle_{\mathrm{L}}^{(A)}}{Z_{\mathrm{L}}^{(A)}}
$$

и определяются через статистическую сумму при фиксированной площади

$$
Z_{\mathrm{L}}^{(A)}=\left(\frac{\pi \gamma\left(b^{2}\right)}{A}\right)^{Q / b} \frac{\Gamma\left(2-b^{2}\right)}{\pi^{3} b^{3} \Gamma\left(b^{2}\right) \Gamma\left(b^{-2}\right)}
$$

и ненормированные корреляции при фиксированной площади

$$
\left\langle V_{a_{1}} \ldots V_{a_{n}}\right\rangle_{\mathrm{L}}^{(A)}=A \int_{\uparrow}\left\langle V_{a_{1}} \ldots V_{a_{n}}\right\rangle_{\mathrm{L}} e^{\mu A} \frac{d \mu}{2 \pi i}=\frac{\left.\left\langle V_{a_{1}} \ldots V_{a_{n}}\right\rangle_{\mathrm{L}}\right|_{\mu \rightarrow A^{-1}}}{\Gamma\left(-b^{-2}-1+b^{-1} \sum_{i=1}^{n} a_{i}\right)} .
$$

Лиувиллевская корреляционная функция в четырехточечном случае может быть построена в почти явном виде как голоморфное-антиголоморфное разложение [12]. Соответствующие формулы приведены в разделе 10, где задача построения коэффициента четвертого порядка обсуждается более детально. Здесь же приведем только четырехточечную функцию полей материи $\langle\varepsilon(x) \varepsilon(0) \varepsilon(1) \varepsilon(\infty)\rangle_{\mathrm{ff}}$. Она составлена из пропагаторов свободных фермионов и в факторизованной форме имеет вид

$$
\langle\varepsilon(x) \varepsilon(0) \varepsilon(1) \varepsilon(\infty)\rangle_{\mathrm{ff}}=F_{\mathrm{ff}}(x) F_{\mathrm{ff}}(\bar{x}),
$$

где

$$
F_{\mathrm{ff}}(x)=\frac{1-x+x^{2}}{x(1-x)}
$$


Отложим анализ более сложного четырехточечного вклада и в качестве предварительного шага ограничимся той информацией теории возмущений, которая имеется в формуле (4.3). На первый взгляд, эта информация слишком бедна для того, чтобы найти вид полной функции скейлинга. Однако мы вскоре увидим, что даже эти ограниченные данные, если их дополнить некоторыми аналитическими свойствами функций скейлинга (которые, в свою очередь, следуют из естественных физических рассуждений) и гипотезой о расположении ее нулей, приводят к неожиданно хорошему описанию этой функции.

\section{5. КРИТИЧЕСКОЕ ПОВЕДЕНИЕ И АСИМПТОТИЧЕСКОЕ ПОВЕДЕНИЕ (ПРИ $T \rightarrow \infty$ )}

Если площадь поверхности становится очень большой по сравнению с характерным масштабом $m^{-\rho}$ возмущений материи, то можно ожидать следующего асимптотического поведения:

$$
Z_{A}(m) \sim A^{Q^{\prime} / b^{\prime}} e^{-\mathcal{E}_{0} A}
$$

Здесь $\mathcal{E}_{0}$ - удельная (на единицу площади) свободная энергия возмущенной материи, взаимодействующей с квантованной гравитацией. Мы также ввели удобный параметр

$$
\rho=(2 s)^{-1} .
$$

Из размерных соображений получим, что

$$
\mathcal{E}_{0}=-m^{2 \rho} f_{0},
$$

где $f_{0}$ - числовая константа, которая в нашей модели зависит от лиувиллевского параметра $b^{2}$. Степенной множитель $A^{Q^{\prime} / b^{\prime}}$ можно оценить, исходя из следующих рассуждений. Почти очевидно, что в нашей модели массивный фермион имеет полную корреляционную длину порядка $m^{-\rho}$, следовательно, на интересных для нас асимптотических масштабах эта длина не влияет на динамику и дает вклад только в локальные величины типа $\mathcal{E}_{0}$. Таким образом, флуктуации больших масштабов обеспечиваются остаточной “материей-наблюдателем” (и духами), поэтому эффективная инфракрасная ТПЛ характеризуется параметрами $b^{\prime}$ и $Q^{\prime}=1 / b^{\prime}+b^{\prime}$. Последний можно найти из баланса центрального заряда в инфракрасной области [1]: справедливы соотношения

$$
1+6\left(Q^{\prime}\right)^{2}+c_{\mathrm{sp}}=26
$$

или

$$
Q^{\prime}=\sqrt{Q^{2}+\frac{1}{12}}
$$

На основе этих физических аргументов можно предположить следующее асимптотическое поведение функции скейлинга $z(t)$ при $t \rightarrow \infty$ :

$$
z(t) \sim \exp \left\{\pi f_{0} t^{\rho}+\rho\left(\frac{Q}{b}-\frac{Q^{\prime}}{b^{\prime}}\right) \ln t+O(1)\right\} .
$$

Заметим, что, как обычно, $\mu_{\text {крит }}=m^{2 \rho} f_{0}$ интерпретируется как положение критической сингулярности в “большой” статистической сумме (3.1). Другими словами, критическое значение $m$ есть $m_{\text {крит }}=\left(\mu / f_{0}\right)^{s}$. 


\section{6. ОСНОВНОЕ ПРЕДПОЛОЖЕНИЕ}

Наше основное предположение состоит в том, что в интересующей нас области $0 \leqslant b^{2} \leqslant 1$ функция скейлинга $z(t)$ имеет асимптотическое поведение (5.6) во всей комплексной плоскости, кроме отрицательной части вещественной оси, где расположены все ее нули. Это ограничение весьма существенно в случае целой функции, и именно оно вместе с некоторыми численными манипуляциями делает наши очень неполные пертурбативные входные данные столь замечательно эффективными.

Асимптотическое поведение $z(t)$ при $t \rightarrow-\infty$ проявляется в результате интерференции двух членов, соответствующих аналитическому продолжению асимптотики (5.6) по и против часовой стрелки:

$$
\begin{aligned}
z(t) & =\exp \left(\pi f_{0} t^{\rho} e^{i \pi \rho}-\left(\frac{1}{2}+\delta\right) \ln e^{i \pi} t+\cdots\right)+\text { к.c. }= \\
& =2 \exp \left(\pi f_{0}(-t)^{\rho} \cos \pi \rho-\left(\frac{1}{2}+\delta\right) \ln (-t)\right) \cos \left(\pi f_{0}(-t)^{\rho} \sin \pi \rho-\pi\left(\frac{1}{2}+\delta\right)\right)
\end{aligned}
$$

Здесь для удобства введено $\delta$ такое, что

$$
\rho\left(\frac{Q}{b}-\frac{Q^{\prime}}{b^{\prime}}\right)=-\frac{1}{2}-\delta .
$$

Асимптотическое положение $n$-го нуля $t_{n}, n=1,2, \ldots$, при $n \rightarrow \infty$ имеет вид

$$
-t_{n}=\left(\frac{n+\delta}{-f_{0} \sin \pi \rho}\right)^{2 s} \text {. }
$$

Если известны нули целой функции, то мы имеем (см. ниже по поводу проблемы сходимости)

$$
z(t)=\prod_{n=1}^{\infty}\left(1-\frac{t}{t_{n}}\right)
$$

и тем самым

$$
\ln z(t)=\sum_{n=1}^{\infty} \ln \left(1-\frac{t}{t_{n}}\right)=t \sum_{n=1}^{\infty} \frac{1}{-t_{n}}-\frac{t^{2}}{2} \sum_{n=1}^{\infty} \frac{1}{\left(-t_{n}\right)^{2}}-\cdots .
$$

Из первых двух членов получим равенства

$$
\begin{gathered}
z_{2}=\sum_{n=1}^{\infty} \frac{1}{-t_{n}} \\
z_{4}-\frac{z_{2}^{2}}{2}=r_{2}=-\frac{1}{2} \sum_{n=1}^{\infty} \frac{1}{\left(-t_{n}\right)^{2}} .
\end{gathered}
$$

В нашем случае произведение (6.4) и сумма (6.6) расходятся, так как порядок $\rho$ нашей функции удовлетворяет неравенству $1 \leqslant \rho<(2-\sqrt{2})^{-1}=1.70711 \ldots$ Можно, однако, показать (см. приложение А), что правило сумм (6.6) остается справедливым, если понимать правую часть этого равенства в терминах дзета-функции 
нулей $t_{n}$. Математически корректное каноническое произведение для функции порядка $\rho<2$ с нулями в точках $t_{n}$ имеет вид

$$
z(t)=e^{z_{2} t} \prod_{n=1}^{\infty}\left(1-\frac{t}{t_{n}}\right) e^{t / t_{n}}
$$

ТАБлица 1. Аппроксимация первого порядка для $f_{0}$ при различных значениях параметра $b^{2}$ и аналитическая оценка для коэффициента разложения $z_{4}$ четвертого порядка, полученная с помощью уравнения (7.5).

\begin{tabular}{|c|c|c|c|c|c|c|}
\hline$b^{2}$ & $s$ & $z_{2}$ & $\delta$ & $-f_{0}$ & $-f_{0}^{\text {(exact) }}$ & $z_{4}^{\text {(est) }}$ \\
\hline 0.05 & 0.488 & -5.26967 & -0.414 & 1.61954 & & 13.8494 \\
\hline 0.10 & 0.475 & -2.7913 & -0.411 & 0.831718 & & 3.85341 \\
\hline 0.15 & 0.463 & -1.9818 & -0.408 & 0.575331 & & 1.91275 \\
\hline 0.20 & 0.450 & -1.59146 & -0.404 & 0.452694 & & 1.20427 \\
\hline 0.25 & 0.438 & -1.37065 & -0.399 & 0.384486 & & 0.863088 \\
\hline 0.30 & 0.427 & -1.23648 & -0.393 & 0.344516 & & 0.670052 \\
\hline 0.35 & 0.415 & -1.15384 & -0.386 & 0.321859 & & 0.547863 \\
\hline 0.40 & 0.404 & -1.10562 & -0.378 & 0.311425 & & 0.462962 \\
\hline 0.45 & 0.393 & -1.08291 & -0.368 & 0.310866 & & 0.398285 \\
\hline 0.50 & 0.382 & -1.08110 & -0.357 & 0.319416 & & 0.343762 \\
\hline 0.55 & 0.372 & -1.09830 & -0.343 & 0.337485 & & 0.292372 \\
\hline 0.60 & 0.362 & -1.13467 & -0.327 & 0.366663 & & 0.2381 \\
\hline 0.65 & 0.352 & -1.19248 & -0.306 & 0.41011 & & 0.174833 \\
\hline 0.70 & 0.342 & -1.27680 & -0.281 & 0.473557 & & 0.094298 \\
\hline 0.75 & 0.333 & -1.39733 & -0.25 & 0.567684 & 0.563124 & -0.016269 \\
\hline 0.80 & 0.325 & -1.57298 & -0.21 & 0.714273 & & -0.18167 \\
\hline 0.85 & 0.316 & -1.84474 & -0.16 & 0.964987 & & -0.462053 \\
\hline 0.90 & 0.308 & -2.32185 & -0.10 & 1.47712 & & -1.05291 \\
\hline 0.95 & 0.300 & -3.45523 & -0.023 & 3.04496 & & -3.04188 \\
\hline 0.99 & 0.294 & -8.75236 & 0.049 & 15.7725 & & -23.4948 \\
\hline
\end{tabular}

\section{7. ЧИСЛЕННЫЙ РАСЧЕТ И ПРИБЛИЖЕНИЯ}

Примем, что асимптотическая формула (6.3) обеспечивает приемлемое приближение для $t_{n}$ даже при малых $n$. Тогда с помощью правила сумм (6.6) можно приблизительно выразить параметр $f_{0}$ в асимптотическом разложении (6.3) через первый коэффициент разложения $z_{2}$ :

$$
z_{2}=\left(f_{0} \sin \pi \rho\right)^{2 s} \sum_{n=1}^{\infty} \frac{1}{(n+\delta)^{2 s}}=\left(f_{0} \sin \pi \rho\right)^{2 s} \zeta(2 s, \delta+1) .
$$

Здесь расходящуюся сумму можно вычислить с помощью аналитического продолжения с использованием $\zeta$-функции Римана. Первая оценка для $f_{0}$ при этом имеет 


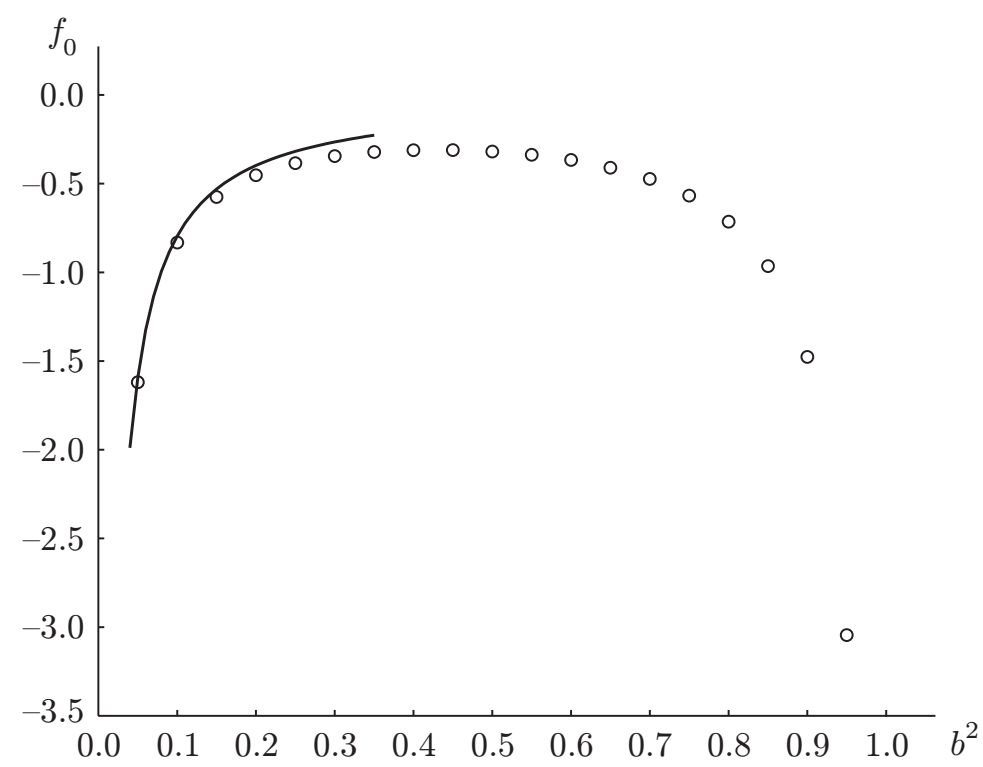

Рис. 1. Аппроксимация первого порядка для $f_{0}$ при различных значениях параметра $b^{2}$ (кружки). Квазиклассический предел представлен непрерывной кривой.

вид

$$
f_{0}=-\frac{1}{\sin \pi \rho}\left(\frac{z_{2}}{\zeta(2 s, \delta+1)}\right)^{\rho} .
$$

Эти оценки при различных значениях параметра $b^{2}$ представлены в табл. 1 и нанесены на рис. 1. Две проверки дают оценку точности приближения. Первая проверка - это сравнение в точке $b^{2}=3 / 4$, которая отвечает чистой модели Изинга (без "материи-наблюдателя") и, как ожидается, допускает точное решение методом матричных моделей. Случайно или нет, но совпадение точных вычислений в матричных моделях (см. раздел 9) с нашими предсказаниями в этой точке оказывается впечатляющим (табл. 1). Другая проверка возможна в классической области $b^{2} \rightarrow 0$. Здесь мы имеем дело с твердой сферой, где эффекты квантовой гравитации подавлены. Удельная энергия вакуума свободного массивного фермиона массы $m$ в плоском классическом фоновом поле имеет вид

$$
\mathcal{E}_{0}=\frac{m^{2}}{4 \pi} \ln m .
$$

Вследствие масштабного свойства (5.3) и соотношения (2.12) мы можем интерпретировать этот факт как следующее ведущее поведение величины $f_{0}\left(b^{2}\right)$ при $b^{2} \rightarrow 0$ :

$$
f_{0}=-\frac{1}{4 \pi b^{2}}+O(1)
$$

Это асимптотическое поведение также изображено на рис. 1. Дальнейшие квазиклассические поправки к вакуумной энергии также могут быть вычислены в 


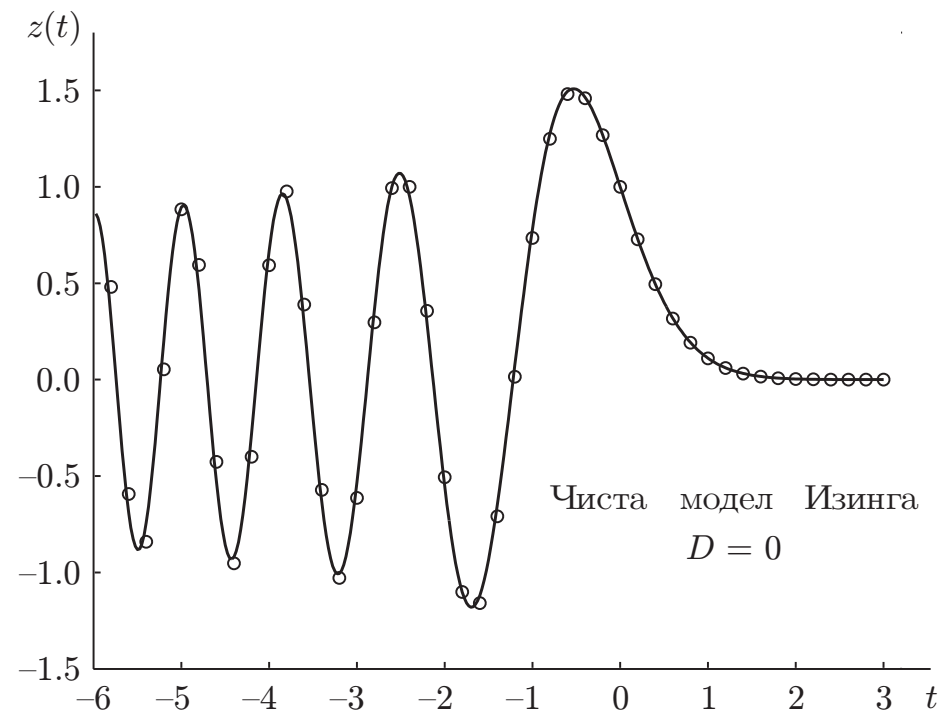

Рис. 2. Функция скейлинга $z(t)$ в точке точного решения $b^{2}=0.75$ : приближение (7.6) (кружки) и точная матрично-модельная функция (8.32) (непрерывная кривая).

теоретико-полевом подходе. Авторы планируют вернуться к этой интересной проблеме в последующих работах.

Из второго правила сумм (6.7) следует оценка для следующего коэффициента разложения $z_{4}$ :

$$
z_{4} \approx z_{4}^{(\mathrm{est})}=\frac{z_{2}^{2}}{2}-\frac{1}{2}\left(-f_{0} \sin \pi \rho\right)^{4 s} \zeta(4 s, \delta+1)
$$

Значения $z_{4}^{\text {(est) }}$ также представлены в табл. 1. Их можно сравнить с результатами прямых вычислений интеграла (4.4). Это было бы еще одной важной проверкой нашей основной гипотезы, а также позволило бы оценить скорость сходимости аналитически-численной процедуры. K сожалению, такое прямое вычисление оказалось технически гораздо более сложным. Его предварительное обсуждение содержится в разделе 10.

Как только получена оценка для параметра $f_{0}$, нули $t_{n}$ могут быть вычислены с помощью асимптотической формулы (6.3). Приближенную функцию скейлинга $z(t)$ при этом можно вычислить в виде (6.8). Другое быстро сходящееся выражение, более удобное для численного расчета, имеет вид

$$
z_{\mathrm{est}}(t)=\exp \left(z_{2} t+\left(z_{4}^{(\mathrm{est})}-\frac{z_{2}^{2}}{2}\right) t^{2}\right) \prod_{n=1}^{\infty}\left(1-\frac{t}{t_{n}}\right) \exp \left(\frac{t}{t_{n}}+\frac{t^{2}}{2 t_{n}^{2}}\right) .
$$

В случае $b^{2}=0.75$ (чистая модель Изинга) эта функция скейлинга изображена на рис. 2, где она сравнивается с точной функцией скейлинга, полученной из матричномодельного решения (см. ниже). 


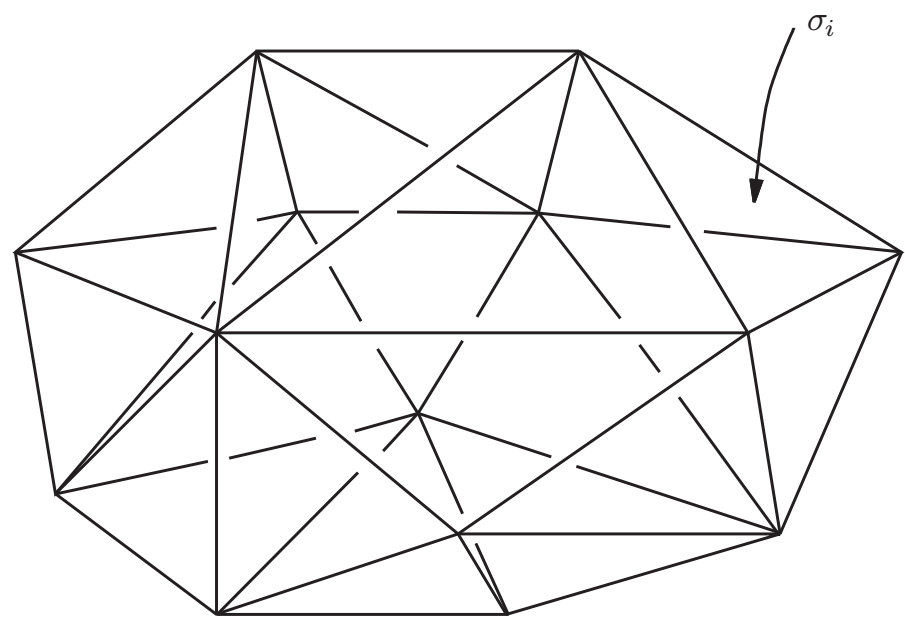

Рис. 3. Образец элемента $\left\{G_{N}\right\}$ при $N=24$. Спиновые переменные Изинга $\sigma_{i}$ ассоциированы с гранями триангуляции.

\section{8. МОДЕЛЬ ИЗИНГА НА СЛУЧАЙНОЙ РЕШЕТКЕ}

Рассмотрим модель дискретной (на случайной решетке) гравитации, которая, как нам кажется, непосредственно связана (в непрерывном пределе) с полевой теорией, развиваемой в предыдущих разделах. Хотя мы и не ожидаем, что непрерывный предел будет существенно зависеть от деталей микроскопического описания решетки, отметим, что здесь мы сформулируем весьма частную систему, а именно модель Изинга на триангулированной случайной решетке. Эта модель в своей основе представляет собой стандартную систему Изинга на случайной решетке, рассмотренную, например, в работах [16], [17]. В этой стандартной формулировке модель оказывается точно решаемой с помощью методов матричных моделей, и в дальнейшем мы используем основные результаты этого подхода. Тем не менее, для дальнейшего сравнения с более общей структурой, введенной в разделе 2 и включающей конформную "материю-наблюдателя", удобно обобщить нашу модель, вводя взаимодействие с $D$-компонентным безмассовым бозоном, или, на языке струнной теории, помещая нашу систему в $D$-мерное конфигурационное пространство. Мы полагаем, что это также сделает свойства данной модели менее примитивными. Можно ожидать, что в непрерывном пределе этот дополнительный решеточный бозон порождает нашу “материю-наблюдателя" по крайней мере в случае топологии сферы, при этом $c_{\mathrm{sp}}=D$.

Возьмем нерегулярную решетку на плоскости (также называемую графом, или триангуляцией), состоящую из $N$ треугольников. Планарность означает, что этот граф может быть нанесен на сферу без самопересечений. Пример такого графа показан на рис. 3. Пусть $\left\{G_{N}\right\}$ представляет собой ансамбль таких топологически различаемых графов. Пронумеруем также треугольники каждого отдельного графа числами $i=1, \ldots, N$ и ассоциируем с каждым из них спиновую переменную $\sigma_{i}= \pm 1$. 
Наконец, пусть $I_{i j}$ представляет собой матрицу инцидентности для соответствующей триангуляции,

$$
I_{i j}= \begin{cases}1, & \text { если треугольники } i \text { и } j \text { имеют общее ребро, } \\ 0 & \text { в противном случае. }\end{cases}
$$

Стандартная модель Изинга отвечает спиновым конфигурациям, распределенным с гиббсовскими весами $W\left[\left\{\sigma_{i}\right\}\right]=\exp \left(-\mathcal{H}\left[\left\{\sigma_{i}\right\}\right]\right)$, где

$$
\mathcal{H}\left[\left\{\sigma_{i}\right\}\right]=K \sum_{i, j} I_{i j} \sigma_{i} \sigma_{j}+H \sum_{i} \sigma_{i},
$$

а $K$ и $H$ - стандартные параметры "температуры" и "магнитного поля". Хотя ненулевое магнитное поле обычно делает физику модели гораздо богаче и интереснее, в данной работе мы ограничимся рассмотрением только случая $H=0$, чтобы избежать дополнительных усложнений. Термодинамическая информация содержится в микроканонической статистической сумме

$$
Z_{N}(K)=N \sum_{\left\{G_{N}\right\}} \operatorname{det}^{-D / 2}\left(\Delta_{i j}^{\text {lat }}\right) \sum_{\left\{\sigma_{i}\right\}} \exp \left(-\mathcal{H}\left[\left\{\sigma_{i}\right\}\right]\right) .
$$

Отметим необычный множитель $N$ в определении статистической суммы; он вводится для упрощения дальнейшего сравнения с непрерывной теорией. Детерминантный множитель отвечает дискретной версии оператора Лапласа

$$
\Delta_{i j}^{\mathrm{lat}}=\left(I_{i j}-3 \delta_{i j}\right)^{\prime} .
$$

В этом множителе появляется новый параметр $D$, играющий роль центрального заряда "материи-наблюдателя". Дискретный оператор Лапласа всегда имеет нулевое собственное число, и штрих в определении (8.4) означает проекцию этого оператора на ненулевое подпространство.

Задача вычисления статистической суммы в указанном виде (8.3) не кажется более простой, чем непрерывный функциональный интеграл по метрикам $g_{a b}$. Для описания непрерывной поверхности следует произвести некий термодинамический предельный переход, при котором размер графа $N$ стремится к бесконечности. В этом пределе непосредственное вычисление статистической суммы кажется весьма сложной задачей. К счастью, при определенном выборе статистических весов существует мощный метод, позволяющий эффективно вычислить термодинамический предел сумм типа (8.3). Это знаменитая техника матричных моделей. Здесь мы не намерены углубляться в детали этой интересной теории, отсылая читателя, например, к обзору [23]. Отметим только, что частный случай модели (8.3) с $D=0$ (чистая МИСР) был точно решен в работах [16] и [17] (см. также работу [24], где рассматривалась в точности наша модель случайных триангуляций с $D=0)$. Из этого точного решения можно почерпнуть некоторые важные особенности ${ }^{4)}$. Мы рассматриваем асимптотику больших $N$, которая только и может быть связана с непрерывной теорией.

\footnotetext{
4) Здесь мы обсуждаем только случай $H=0$, хотя и в случае ненулевого магнитного поля модель также оказывается точно решаемой.
} 
1. При общих значениях температурного параметра $K$ асимптотическое поведение статистической суммы при $N \rightarrow \infty$ имеет вид

$$
Z_{N}(K) \sim \mathcal{Z}(K) N^{-5 / 2} e^{-E(K) N} .
$$

Здесь $E(K)$ - удельная (на один треугольник) свободная энергия. Эта функция неуниверсальна и зависит от деталей МСР. Явный вид этой функции в рассматриваемом весьма частном случае можно найти в работе [24]. Напротив, степенная зависимость предэкспоненциального множителя $N^{-5 / 2}$ оказывается универсальной, и показатель $-5 / 2$ известен как критический индекс “чистой гравитации"5).

2. Критичность, как и в стандартной модели Изинга, имеет место при определенном значении $K=K_{\text {с }}$ (критической температуре), которое опять-таки зависит от микроскопических деталей модели. В нашей частной триангулированной системе имеем [24]

$$
K_{\mathrm{c}}=\frac{1}{2} \ln \frac{23}{108} .
$$

Асимптотическое поведение (8.5) при этом видоизменяется:

$$
Z_{N}\left(K_{\mathrm{c}}\right) \sim \mathcal{Z}_{\mathrm{c}} N^{-7 / 3} e^{-E\left(K_{\mathrm{c}}\right) N}
$$

В степенном поведении предэкспоненциального множителя возникает показатель $-7 / 3$, известный как критический индекс "гравитационной модели Изинга". В частности, это изменение асимптотического режима означает то, что функция $\mathcal{Z}(K)$ сингулярна при $K=K_{\mathrm{c}}$. В любом случае $\mathcal{Z}_{\mathrm{c}} \neq \mathcal{Z}\left(K_{\mathrm{c}}\right)$.

3. Критическое масштабное поведение по $K$ вблизи критической точки, как ожидается, должно быть универсальным и наблюдаемым в теоретико-полевых приложениях. Пусть

$$
\tau=\frac{K-K_{\mathrm{c}}}{K_{\mathrm{c}}}, \quad|\tau| \ll 1 .
$$

В этой области удобно ввести параметр $N_{\mathrm{c}}$ "корреляционного размера" на решетке.

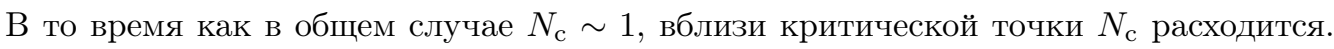
Точное решение МИСР показывает, что

$$
N_{\mathrm{c}} \sim \frac{L_{0}}{|\tau|^{3}} \gg 1
$$

где $L_{0}$ - некоторая (неуниверсальная) постоянная, зависящая от выбора масштаба. Рассматривая асимптотическое поведение $Z_{N}(K)$ в пределе больших $N$, выделим следующие области.

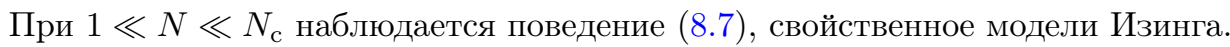

При $N \gg N_{\text {с }}$ изинговские спины коррелируют локально, давая вклад только в

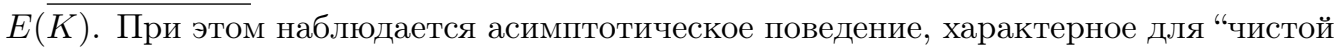
гравитации" (8.5), с некоторым вкладом в $E(K)=E_{\mathrm{reg}}(K)+E_{\mathrm{sing}}(\tau)$, сингулярным

\footnotetext{
5) Он отличается от знаменитого показателя $-7 / 2$, полученного в работе [15], только из-за дополнительного множителя $N$ в определении (8.3).
} 
по $\tau$. Эта сингулярность вызвана дальнодействующими (по сравнению с шагом решетки) корреляциями изинговских спинов:

$$
Z_{N}(K) \sim \mathcal{Z}\left(K_{\mathrm{c}}\right) N^{-5 / 2} e^{-E_{\mathrm{reg}}(K) N-E_{\mathrm{sing}}(\tau) N} .
$$

Сингулярный вклад $E_{\text {sing }}(\tau)$ в точно решаемую МИСР имеет вид

$$
E_{\text {sing }}(\tau)=e_{0}|\tau|^{3},
$$

где амплитуда критической сингулярности $e_{0}$ снова зависит от выбора масштаба.

При $N \sim N_{\mathrm{c}}$ возникает критическая функция скейлинга $F_{\text {sphere }}(y)$ в соотношении

$$
Z_{N}(K) \sim F_{\text {sphere }}\left(\frac{N}{N_{\mathrm{c}}}\right) N^{-7 / 3} e^{-E_{\text {reg }}(K) N} .
$$

Эта функция имеет следующие асимптотические свойства при малых и больших значениях аргумента:

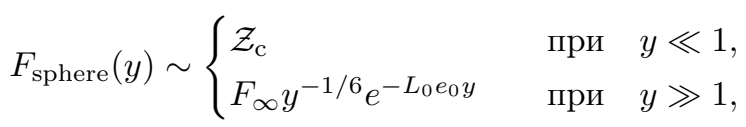

где, очевидно,

$$
F_{\infty}=\mathcal{Z}\left(K_{\mathrm{c}}\right) N_{\mathrm{c}}^{-1 / 6}
$$

Более того, точное решение матричной модели задает функцию $F_{\text {sphere }}(y)$ MИСР в явном виде. Перед тем как это обсудить, предположим, что имеет место естественное обобщение приведенных масштабирующих соотношений в модели (8.3) при $-\infty<D \leqslant 1 / 2$. Эту модель при $D \neq 0$ нам представляется естественным назвать обобщенной моделью Изинга на случайной решетке (ОМИСР). В рамках предложенного нами соотношения с непрерывной квантовой гравитацией, описанного в разделе 2 , необходимо заменить общее асимптотическое поведение (8.5) на

$$
Z_{N}(K, D) \sim \mathcal{Z}(K, D) N^{-d_{\mathrm{g}}(D)} e^{-E(K, D) N},
$$

где мы ожидаем встретить уже знакомое $c_{\mathrm{sp}}=D$ масштабное преобразование Книжника-Полякова-Замолодчикова $[8]^{6)}$ :

$$
d_{\mathrm{g}}(D)=\frac{1}{12}(25-D+\sqrt{(25-D)(1-D)}) .
$$

В общем случае ожидается наличие критической точки $K=K_{\mathrm{c}}(D)$, в которой асимптотическое поведение приобретает иной характер:

$$
Z_{N}\left(K_{\mathrm{c}}, D\right) \sim \mathcal{Z}_{\mathrm{c}}(D) N^{-d_{\mathrm{c}}(D)} e^{-E\left(K_{\mathrm{c}}, D\right) N} .
$$

Теперь

$$
d_{\mathrm{c}}(D)=\frac{1}{24}(49-2 D+\sqrt{(49-2 D)(1-2 D)}) .
$$

6)Этот показатель связан со знаменитым индексом $\gamma_{\text {string }}$ [4] с помощью соотношения $d_{\mathrm{g}}(D)=$ $2-\gamma_{\text {string. }}$. 
Величина $E(K, D)$ может быть снова интерпретирована как удельная (на один треугольник) свободная энергия бесконечной системы. При $K=K_{\text {с }}$ она имеет сингулярность:

$$
E(K, D)=E_{\mathrm{reg}}(K, D)+e_{0}(D)|\tau|^{2 \rho(D)},
$$

где

$$
\rho(D)=\frac{(\sqrt{49-2 D}-\sqrt{1-2 D})(\sqrt{49-2 D}+\sqrt{25-2 D})}{48},
$$

величина $\tau$ определена в формуле $(8.8)$ и $e_{0}(D)$ - амплитуда критической сингулярности. Наконец, в области скейлинга $|\tau| \ll 1$, корреляционный размер подчиняется соотношению

$$
N_{\mathrm{c}}(D) \sim L_{0}(D)|\tau|^{-2 \rho(D)}
$$

и становится большим по сравнению с характерным размером решетки. В этой

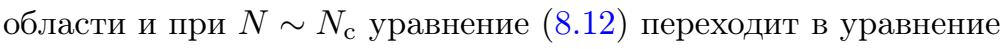

$$
Z_{N}(K, D) \sim F_{\text {sphere }}\left(\frac{N}{N_{\mathrm{c}}}, D\right) N^{-d_{\mathrm{c}}(D)} e^{-E_{\mathrm{reg}}(K, D) N},
$$

где функция скейлинга теперь зависит от $D$ и

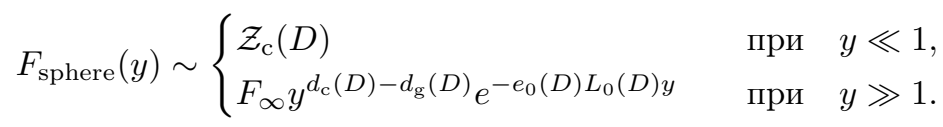

Заметим, что мы ожидаем определенной симметрии $\tau \rightarrow-\tau$ для сингулярной части. Это можно предвидеть на основании свойства дуальности модели Изинга. Эта симметрия также имеет место и в случае случайных решеток, при отображении модели на триангулированной решетке на дуальную модель на графе теории $\phi^{3}$. Разумеется, эти соображения в большой мере основываются на универсальности критического поведения, и из них следует, что симметрия характерна только для масштабно-инвариантных характеристик. Например, масштабно-зависимые множители типа $L_{0}(D)$ и $e_{0}(D)$, равно как и общая нормировка функции скейлинга, могут быть существенно различными в высоко- и низкотемпературной фазах $\tau<0$ и $\tau>0$, соответственно. Мы не отмечали это обстоятельство выше с целью сделать уравнения более наглядными.

Легко связать все эти масштабные характеристики с наблюдаемыми теоретикополевой задачи, обсуждаемой в разделе 2. Разумеется, лиувиллевские параметры $b$ и $a$ в лагранжиане (2.1) имеют вид

$$
b=\sqrt{\frac{49-2 D}{48}}-\sqrt{\frac{1-2 D}{48}}, \quad a=\sqrt{\frac{49-2 D}{48}}-\sqrt{\frac{25-2 D}{48}} .
$$

Функция скейлинга $z(t)$, определенная в уравнении (3.11), представляет собой с точностью до общей нормировки и нормировки аргумента не что иное, как $F_{\text {sphere }}(y, D)$ в ОМИСР. Более точно,

$$
f_{\text {sphere }}(y, D)=\frac{F_{\text {sphere }}(y, D)}{F_{\text {sphere }}(0, D)}=z(t),
$$


где $y$ пропорционален $t^{\rho}$, причем коэффициент пропорциональности зависит от выбора масштаба (или от точного определения величины $N_{\text {c }}$ ). Чтобы быть несколько более точными, введем размерные параметры $a_{0}$ и $m_{0}$, которые связывают величины $N$ и $\tau$ микроскопической модели с площадью $A$ и константой связи $m$ :

$$
A=a_{0} N, \quad m=m_{0} \tau .
$$

Мы также однозначно фиксируем корреляционный размер поверхности с помощью соотношения

$$
A_{\mathrm{c}}=\pi m^{-2 \rho},
$$

при этом

$$
\begin{aligned}
y & =\frac{A}{A_{\mathrm{c}}}=t^{\rho}, \\
L_{0} & =\frac{\pi}{a_{0} m_{0}^{2 \rho}} .
\end{aligned}
$$

В частности, амплитуда $e_{0}(D)$ в уравнении (8.19) связана с универсальным параметром $f_{0}$ в уравнении (5.3):

$$
L_{0}(D) e_{0}(D)=\pi f_{0}
$$

Как только нормировка фиксирована, мы можем сравнить нормированную функцию скейлинга ОМИСР $f_{\text {sphere }}(y, D)$ с нашими предсказаниями, полученными в рамках теории возмущений $(3.11),(4.3)$, т.е. с выражением

$$
f_{\text {sphere }}(y, D)=1+z_{2} y^{2 s}+z_{4} y^{4 s}+\cdots,
$$

равно как и с асимптотическим поведением, в основном контролируемым параметром $f_{0}$ из табл. 1. В частности, в точно решаемом случае $D=0$ эта функция скейлинга выписывается в явном виде (см. детали в следующем разделе):

$$
f_{\text {sphere }}(y, 0)=3^{2 / 3} \Gamma\left(\frac{2}{3}\right) \operatorname{Ai}\left(\frac{3^{2 / 3} l_{\mathrm{eg}}^{2}}{4} y^{2 / 3}\right),
$$

где $l_{\text {eg }}$ есть числовая константа,

$$
l_{\mathrm{eg}}=2 \gamma(1 / 3) \gamma^{2 / 3}(3 / 4)=1.91987 \ldots,
$$

связанная с нашим частным выбором $N_{\mathrm{c}}$, а $\mathrm{Ai}(x)$ - функция Эйри,

$$
\operatorname{Ai}(x)=\frac{1}{3^{2 / 3}} \sum_{n=0}^{\infty} \frac{\left(-3^{1 / 3} x\right)^{n}}{n ! \Gamma(2 / 3-n / 3)} .
$$

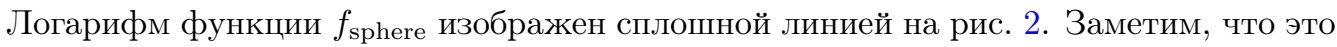
разложение функции Эйри в степенной ряд предсказывает, что $z_{4}=0$ в точке чистой модели Изинга, т.е. при $b^{2}=0.75$. В последнем разделе данной статьи мы собира- 


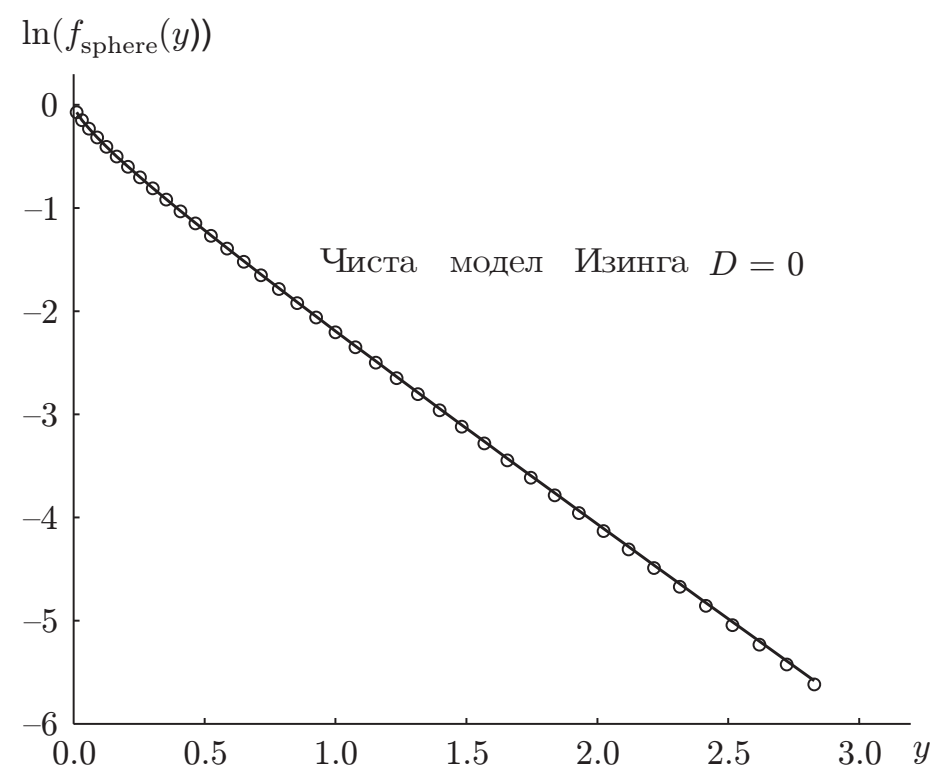

Рис. 4. Логарифмический график функции $f_{\text {sphere }}(y)$ в случае чистой модели Изинга $D=0$. Кружки - приближение, непрерывная линия - точная матрично-модельная функция.

емся подтвердить это заключение прямым вычислением интеграла лиувиллевской гравитации (4.4).

В следующем разделе обсуждается точное решение матричной модели в непрерывном пределе. Здесь мы отметим, что обобщенные модели на случайных решетках при общих значениях $D \leqslant 1 / 2$ представляют собой достаточно интересные статистические модели динамических решеток. Хотя наш теоретико-полевой подход содержит много не слишком хорошо обоснованных предположений и гипотез, из него следуют вполне определенные предсказания для измеримых наблюдаемых в ОМИСР как в случае $D=0$, так и при $D \neq 0$, в том числе в тех случаях, когда знаменитые матричные модели (часто принимаемые в качестве замены полевой теории) не могут дать никаких определенных ответов. Является ли это случайным численным совпадением или нет, но наши результаты, представленные на рис. 2, обеспечивают удивительно хорошее и детальное описание точной функции скейлинга матричной модели. На рис. 4 аналогичное сравнение проводится для $f_{\text {sphere }}(y, 0)$ при имеющих физический смысл положительных значениях $t$ и достаточно больших значениях $y$. В этом случае явно проявляется экспоненциальное асимптотическое поведение (5.1). Заметим, что на этом графике (в логарифмическом масштабе) прослеживается убывание функции $f_{\text {sphere }}(y, 0)$ по крайней мере на протяжении трех порядков ее величины. На рис. 5 мы также показываем наше приближение функции скейлинга $f_{\text {sphere }}(y, D)$ при $D=-2 \quad\left(b^{2}=0.530049 \ldots\right)$ и при $D=-20$ $\left(b^{2}=0.191377 \ldots\right)$. 


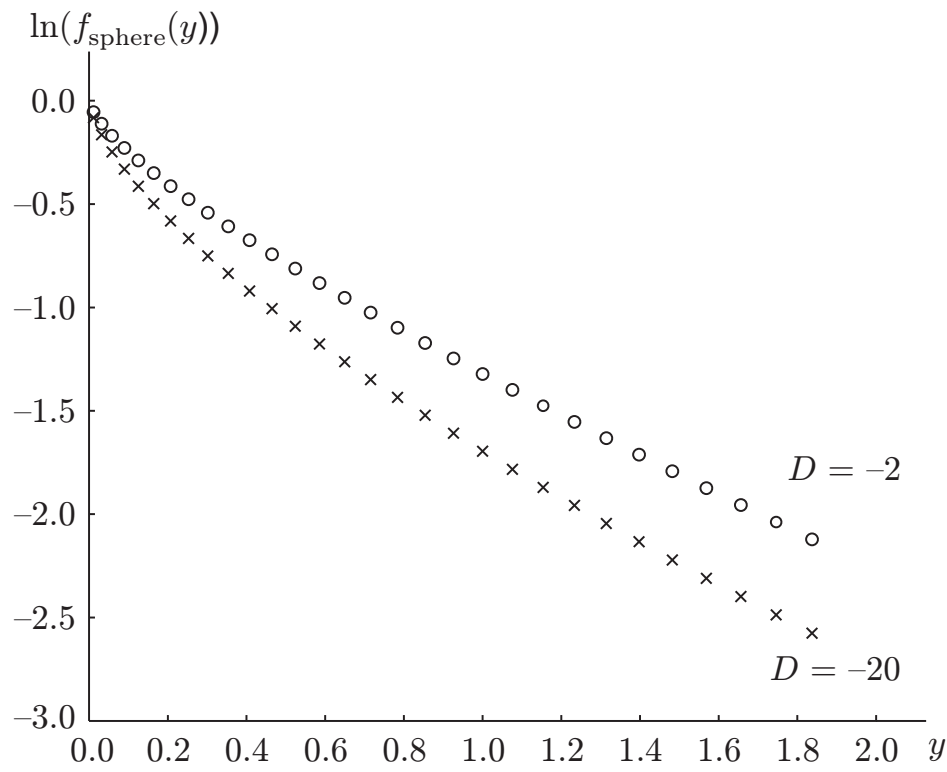

Рис. 5. Приближение для функций скейлинга $f_{\text {sphere }}(y, D)$ при $D=-2$ и $D=-20$.

\section{9. МИНИМАЛЬНАЯ ГРАВИТАЦИЯ (ЧИСТАЯ МОДЕЛЬ ИЗИНГА)}

Рассмотрим точку $b^{2}=3 / 4$ (или же, на языке "материи-наблюдателя", случай $\left.c_{\mathrm{sp}}=D=0\right)$. Этот случай, как ожидается, описывается двухматричной моделью Казакова [16], [17]. В двойном скейлинговом пределе этой матричной модели статистическая сумма $Z(T, x)$ для рода ноль задается уравнением ${ }^{7)}$

$$
u(T, x)=Z_{x x}(T, x),
$$

где $u(T, x)$ - решение следующего (перенормированного) алгебраического уравнения [16]:

$$
x=u^{3}-\frac{3}{4} T^{2} u .
$$

В этом уравнении $x$ играет роль космологической постоянной, а $T$ отвечает отклонению температуры системы Изинга от критического значения. Точные соотношения между параметрами $T$ и $x$ в этом разделе (а также соответственно между $\tau$ и действием, сопряженным величине $N$, в предыдущем разделе) зависят от микроскопической реализации МИСР. В частной формулировке, приведенной в разделе 8 , эти параметры связаны между собой явными формулами из работы [24].

7)В этом месте мы, как и другие авторы, пишущие про матричные модели, достаточно вольно обращаемся с нормировками статистической суммы и структурных констант. В этом подходе имеют значение только масштабно-инвариантные комбинации. 
Из формул (9.1) и (9.2) можно получить разложение по $T^{2}$ :

$$
\begin{aligned}
Z(T, x) & =-\frac{x^{7 / 3}}{3} \sum \frac{\Gamma(2 n / 3-7 / 3)}{n ! \Gamma(2 / 3-n / 3)}\left(-\frac{3 T^{2}}{4 x^{2 / 3}}\right)^{n}= \\
& =\frac{9 x^{7 / 3}}{28}+\frac{9 T^{2} x^{5 / 3}}{40}+\frac{3 T^{6} x^{1 / 3}}{128}+\frac{3 T^{8}}{1024 x^{1 / 3}}+\cdots .
\end{aligned}
$$

Статистическая сумма при фиксированной площади $а$ (мы обозначаем эту площадь символом $a$ вместо $A$, поскольку нормировка сопряженной космологической постоянной $x$ отличается от нормировки для $\mu$ ) имеет вид

$$
Z_{a}(T)=a \int_{\uparrow} Z(T, x) e^{a x} \frac{d x}{2 \pi i},
$$

где контур $\uparrow$ выбран так же, как и в формуле (3.7). Это выражение может быть преобразовано к виду

$$
Z_{a}(T)=-\frac{1}{3} a^{-7 / 3} \int_{\uparrow} \exp \left(v^{2}-\frac{3}{4} a^{2 / 3} T^{2} v^{2 / 3}\right) \frac{d v}{\pi i v^{1 / 3}}
$$

и затем выражено через функции Эйри:

$$
Z_{a}(T)=-\frac{a^{-7 / 3}}{3^{1 / 3}} \operatorname{Ai}\left(\frac{(3 a)^{2 / 3} T^{2}}{4}\right) .
$$

Легко вывести соотношение между масштабами

$$
T a^{1 / 3}=l_{\mathrm{eg}} m\left(\frac{A}{\pi}\right)^{1 / 3}
$$

$\left(l_{\mathrm{eg}}\right.$ взято из уравнения $\left.(8.33)\right)$. Достаточно сравнить члены разложений порядка $T^{2}$ в формуле (9.3) и порядка $z_{2}$ в формуле (4.3). В итоге выражение (9.6) сведется к

$$
Z_{a}(T)=-3^{-1 / 3} A^{-7 / 3} \operatorname{Ai}\left(\left(m\left(\frac{3 A}{\pi}\right)^{1 / 3} \frac{l_{\mathrm{eg}}}{2}\right)^{2}\right) .
$$

С точностью до нормировки это выражение эквивалентно (8.32). В частности,

$$
Z_{a} \sim \exp \left(-l_{\mathrm{eg}}^{3} m^{3} \frac{A}{4 \pi}\right)
$$

Из этого асимптотического выражения получим

$$
-f_{0}^{(\text {exact })}=\frac{l_{\text {eg }}^{3}}{4 \pi}=\frac{2 \gamma^{3}(1 / 3)}{\pi \gamma^{2}(1 / 4)}=0.563124 \ldots,
$$

т.е. число, приведенное в шестой колонке табл. 1. 


\section{0. ЧЕТЫРЕХТОЧЕЧНЫЙ ИНТЕГРАЛ}

Рассмотрим (на предварительной основе) проблему вычисления поправки четвертого порядка (4.4). Составляющая полей материи в подынтегральном выражении достаточно проста, см. формулу (4.8). Явное выражение для лиувиллевской четырехточечной функции можно найти в работе [12]. Здесь нам понадобится только "симметричный" вариант этой функции, в котором все четыре внешние размерности совпадают (и равны 1/2). Из вычислительных соображений удобно свести голоморфно-антиголоморфный (спектральный) интеграл к виду [1]

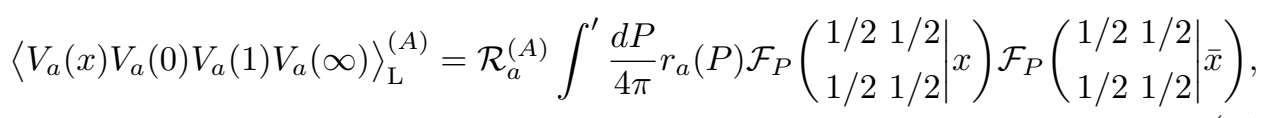

где общий множитель равен

$$
\mathcal{R}_{a}^{(A)}=\frac{\left(\pi \gamma\left(b^{2}\right) b^{2-2 b^{2}} A^{-1}\right)^{(Q-4 a) / b}}{\pi^{2} \Gamma\left(-b^{-2}-1+4 b^{-1} a\right)} \frac{\Upsilon_{b}^{4}(b) \Upsilon_{b}^{4}(2 a)}{\Upsilon_{b}^{4}(2 a-Q / 2)},
$$

а весовая функция есть

$$
r_{a}(P)=\frac{\pi^{2} \Upsilon_{b}(2 i P) \Upsilon_{b}(-2 i P) \Upsilon_{b}^{4}(2 a-Q / 2)}{\Upsilon_{b}^{2}(b) \Upsilon_{b}^{2}(2 a-Q / 2-i P) \Upsilon_{b}^{2}(2 a-Q / 2+i P) \Upsilon_{b}^{2}(Q / 2-i P) \Upsilon_{b}^{2}(Q / 2+i P)}
$$

Штрих возле знака интеграла в выражении (10.1) обозначает возможные дискретные члены. Функция общего конформного блока

$$
\left.\mathcal{F}_{P}\left(\begin{array}{cc}
\Delta_{1} & \Delta_{3} \\
\Delta_{2} & \Delta_{4}
\end{array} \mid x\right)=\sum_{x_{2}(=0)}^{x_{1}(=x)}\right\rangle_{\Delta_{2}}^{\Delta_{1}} x_{4}(=\infty) \backslash \Delta_{4}
$$

может быть эффективно вычислена с помощью рекурсивной процедуры, приведенной в работах [25]. В данной работе нас интересует частный случай $\Delta_{1}=\Delta_{2}=$ $\Delta_{3}=\Delta_{4}=1 / 2$.

Легко видеть, что в нашем примере теории свободного фермиона в правой части уравнения (10.1) присутствует по крайней мере один дискретный член, поскольку всегда выполнено условие $2 a<Q / 2$. Этот дискретный член расположен в точках $P= \pm i(Q / 2-2 a)$. Более того, если $b^{2}<b_{1}^{2}$, где $b_{1}^{2}=(\sqrt{8 / 3}+1)^{-1} \approx 0.38$, то в точках $P= \pm i(Q / 2-2 a-b)$ возникает второй дискретный член и т.д. Таким образом, для $b^{2}$, меньших $b_{1}^{2}$, появляется все больше и больше дискретных членов. Именно эти члены оказываются значимыми в классическом пределе $b^{2} \rightarrow 0$. Все эти усложнения затрудняют непосредственный численный расчет интеграла (4.4). Общая проблема исследована более подробно в работе [26], в которой развита соответствующая техника численного интегрирования. Здесь мы ограничимся предварительными выражениями в простейшем случае $b^{2}>b_{1}^{2}$, в котором возникает только один дискретный 
член. Не так уж сложно выделить этот член в явном виде:

$$
\left\langle V_{a}(x) V_{a}(0) V_{a}(1) V_{a}(\infty)\right\rangle_{\mathrm{L}}^{(A)}=\text { дискретный член }+\mathcal{R}_{a}^{(A)} \int r_{a}(P) \mathcal{F}_{P}(x) \mathcal{F}_{P}(\bar{x}) \frac{d P}{4 \pi},
$$

где второе слагаемое представляет собой обычный интеграл по вещественной оси значений параметра $P$. Весовая функция $r_{a}(P)$ допускает следующее удобное интегральное представление, которое всегда сходится при $1>b^{2}>0$ :

$$
\begin{aligned}
r_{a}(P)= & \frac{\operatorname{sh}\left(2 \pi b^{-1}\right) P \operatorname{sh}(2 \pi b P) \gamma^{4}\left(3 / 2+b^{-2} / 2-2 a b^{-1}\right)}{\gamma^{2}\left(3 / 2+b^{-2} / 2-2 a b^{-1}+i b^{-1} P\right) \gamma^{2}\left(3 / 2-b^{-2} / 2-2 a b^{-1}-i b^{-1} P\right)} \times \\
& \times \exp \left(-8 \int_{0}^{\infty} \frac{d t}{t} \frac{\sin ^{2}(P t)\left(\operatorname{ch}^{2}(b t-2 a t)-e^{-Q t} \cos ^{2}(P t)\right)}{\operatorname{sh}(b t) \operatorname{sh}\left(b^{-1} t\right)}\right) .
\end{aligned}
$$

В симметричном случае равных внешних размерностей дискретный член обязан своим появлением двойному полюсу функции $r_{a}(P)$ и, тем самым, оказывается в некотором смысле "логарифмическим"8):

$$
\begin{aligned}
\text { дискретный член }= & \left(\frac{\pi \gamma\left(b^{2}\right)}{A}\right)^{(Q-4 a) / b} \frac{\gamma\left(4 a b-b^{2}\right)}{b^{2} \Gamma\left(2-4 a b^{-1}+b^{-2}\right)}\left|\mathcal{F}_{i(Q / 2-2 a)}(x)\right|^{2} \times \\
& \times\left(2 \operatorname{Re} f_{i(Q / 2-2 a)}(x)-2 v_{b}(4 a)+4 v_{b}(2 a)-v_{b}^{\prime \prime}\right),
\end{aligned}
$$

где мы ввели обозначения

$$
\begin{gathered}
v_{b}(x)=\frac{d}{d x} \ln \Upsilon_{b}(x), \\
v_{b}^{\prime \prime}=\frac{\Upsilon_{b}^{\prime \prime}(0)}{\Upsilon_{b}^{\prime}(0)}=2\left(2 b \ln b-2 \Gamma^{\prime}(1) b+v_{b}(b)\right), \\
f_{P}(x)=i \frac{d}{d P} \ln \mathcal{F}_{P}(x) .
\end{gathered}
$$

Используя эти выражения, мы теперь можем численно найти интеграл в формуле (4.4). Коэффициент разложения $z_{4}$ при фиксированной площади задается отношением

$$
z_{4}=\frac{\int G_{\mathrm{ff}}(x, \bar{x}) G_{\mathrm{L}}^{(A=\pi)}(x, \bar{x}) d^{2} x}{24(2 \pi)^{4} Z_{\mathrm{L}}^{(A=\pi)}} .
$$

Из формулы (4.6) имеем

$$
Z_{\mathrm{L}}^{(A=\pi)}=\frac{\gamma^{Q / b}\left(b^{2}\right) \Gamma\left(2-b^{2}\right)}{\pi^{3} b^{3} \Gamma\left(b^{2}\right) \Gamma\left(b^{-2}\right)} .
$$

Эти вычисления были произведены с помощью техники численного расчета, развитой в работе [26]. В табл. 2 отдельно представлены вклады дискретного $\left(z_{4}^{(\text {disc) }}\right)$ и интегрального $\left(z_{4}^{(\mathrm{int})}\right)$ членов, а также их суммарный вклад $\left(z_{4}^{(\mathrm{num})}\right)$ и оценка $(7.5)$. Более детальный анализ четырехточечного корреляционного числа и его вкладов в

\footnotetext{
8) Исследователи логарифмических КТП могут быть спокойны - это обычное вырождение.
} 
ТАБлицА 2. Результаты численного интегрирования для “дискретной” и “интегральной" частей коэффициента разложения четвертого порядка $z_{4}$ в сравнении с оценкой, вытекающей из уравнения (7.5)

\begin{tabular}{|c|c|c|c|c|}
\hline$b^{2}$ & $z_{4}^{\text {(disc) }}$ & $z_{4}^{\text {(int) }}$ & $z_{4}^{\text {(num) }}$ & $z_{4}^{\text {(est) }}$ \\
\hline 0.40 & 0.442804 & 0.0116879 & 0.454492 & 0.462962 \\
\hline 0.45 & 0.377819 & 0.0140653 & 0.391885 & 0.398285 \\
\hline 0.50 & 0.323998 & 0.0164891 & 0.340487 & 0.343762 \\
\hline 0.55 & 0.274771 & 0.0186348 & 0.293406 & 0.292372 \\
\hline 0.60 & 0.224707 & 0.0198837 & 0.244591 & 0.238100 \\
\hline 0.65 & 0.168093 & 0.0190699 & 0.187163 & 0.174833 \\
\hline 0.70 & 0.0973206 & 0.0139516 & 0.111272 & 0.094298 \\
\hline 0.75 & $-2 . \times 10^{-8}$ & 0.0000000 & $-2 . \times 10^{-8}$ & -0.016269 \\
\hline 0.80 & -0.147962 & -0.032752 & -0.180715 & -0.18167 \\
\hline 0.85 & -0.403419 & -0.109586 & -0.513006 & -0.462053 \\
\hline 0.90 & -0.943565 & -0.312733 & -1.25630 & -1.05291 \\
\hline 0.95 & -2.72197 & -1.09636 & -3.81834 & -3.04188 \\
\hline 0.99 & -20.4533 & -9.61235 & -30.0656 & -23.4948 \\
\hline
\end{tabular}

функцию скейлинга и в параметр свободной энергии $f_{0}$ мы намерены представить в отдельной публикации.

В случае $b^{2}=0.75$ ("матричной модели") ситуация существенно упрощается. Вопервых, легко показать, что дискретный член оказывается сингулярен в окрестности этой точки. В ней $4 a=Q-b$, и мы оказываемся в резонансе первого порядка. Тем не менее в корреляционной функции (4.7) при фиксированной площади соответствующий полюс сокращается с полюсом гамма-функции в знаменателе (4.7) и остается конечное выражение. Более того, этот знаменатель обращает в нуль остаточный интегральный член, и единственный ненулевой вклад дается дискретным членом. В этом случае нетрудно явно вычислить лиувиллевскую четырехточечную функцию при фиксированной площади (см., например, [1]):

$$
\left\langle V_{a}(x) V_{a}(0) V_{a}(1) V_{a}(\infty)\right\rangle_{\mathrm{L}}^{(A)}=\frac{4[K(x) K(1-\bar{x})+K(\bar{x}) K(1-x)]}{b A[x \bar{x}(1-x)(1-\bar{x})]^{1 / 6}},
$$

где

$$
K(x)=\frac{1}{2} \int[t(1-t)(1-x t)]^{-1 / 2} d t
$$

есть полный эллиптический интеграл первого рода. Таким образом, с четырехточечной функцией полей материи (4.8) наша задача сведется к вычислению интеграла

$$
I\left(\frac{1}{6}\right)=\int \frac{\left(1-x+x^{2}\right)\left(1-\bar{x}+\bar{x}^{2}\right)}{x \bar{x}(1-x)(1-\bar{x})} \frac{K(x) K(1-\bar{x})+K(\bar{x}) K(1-x)}{[x \bar{x}(1-x)(1-\bar{x})]^{1 / 6}} d^{2} x .
$$

Этот интеграл расходится, и нам необходимо найти его конечную часть. На самом деле, мы намерены продемонстрировать, что эта конечная часть обращается в нуль. 
Если бы это было не так, то соответствующий резонансный вклад проявлялся бы как член четвертого порядка вида $m^{4} \mu \ln \mu$ в большой статистической сумме (3.3). Но такие логарифмические поправки никогда не появляются в матричных моделях (при вычислениях, схожих с расчетом по методу среднего поля). Если мы надеемся, что точка $b^{2}=3 / 4$ в самом деле имеет отношение к точно решаемой матричной модели, то естественно ожидать, что логарифмический член будет сокращаться в результате обращения интеграла (10.14) в нуль. И в самом деле, оценки для коэффициента четвертого порядка $z_{4}$ с помощью численного интегрирования выражения (10.10) (см. табл. 2), равно как и с помощью правила сумм (6.7), дают весьма близкий к нулю ответ, как можно видеть из табл. 1. В приложении Б это сокращение также показывается аналитически.

\section{Дзета-регуляризация}

ПРИЛОЖЕНИЕ А

Пусть

$$
z(t)=\sum_{n=0}^{\infty} z_{2 n} t^{n}
$$

- целая функция порядка $\rho, t_{n}, n=1,2, \ldots,-$ ее нули, которые предполагаются вещественными и отрицательными. Мы нормируем эту функцию так, что $z(0)=1$. Для упрощения рассмотрения предположим, что $1<\rho<2$, хотя наши рассуждения непосредственно обобщаются на произвольный конечный порядок. Сходящееся каноническое произведение имеет вид

$$
z_{\text {can }}(t)=\prod_{n=1}^{\infty}\left(1-\frac{t}{t_{n}}\right) \exp \left(\frac{t}{t_{n}}\right),
$$

и, очевидно,

$$
z(t)=e^{z_{2} t} z_{\text {can }}(t)
$$

Дзета-функция нулей задается с помощью ряда ${ }^{9)}$ :

$$
\zeta_{z}(s)=\sum_{n=1}^{\infty} \frac{1}{\left(-t_{n}\right)^{s}},
$$

который сходится при $\operatorname{Re} s>\rho$, а при остальных значениях понимается как аналитическое продолжение. При $2>\operatorname{Re} s>\rho$ этот ряд можно определить через каноническое произведение:

$$
\zeta_{z}(s)=\frac{\sin \pi s}{\pi} \int_{0}^{\infty} t^{-s} \frac{d \ln z_{\mathrm{can}}(t)}{d t} d t
$$

(здесь необходимо выбрать $z_{\text {сап }}(t)$ вместо $z(t)$, чтобы избежать расходимости при $t=0)$. И наоборот,

$$
\ln z_{\mathrm{can}}(t)=\int_{\uparrow} \frac{\pi \zeta_{z}(s)}{\sin \pi s} t^{s} \frac{d s}{2 i \pi s},
$$

\footnotetext{
9) Лучше не путать параметр $s$ в этом выражении с величиной $a b^{-1}$ в основном тексте статьи.
} 
где контур интегрирования $\uparrow$ проходит вдоль мнимой оси справа от полюса при $s=\rho$ и слева от полюса при $s=2$. В то же время полюс в точке $s=1$ дает вклад в асимптотическое выражение для $\ln z_{\text {сап }}(t)$ при $t \rightarrow \infty$. Сравнивая с (А.3), найдем, что

$$
z_{2}=\zeta_{z}(1)
$$

Расходящуюся сумму в формуле (6.6) следует понимать как аналитическое продолжение (А.4).

\section{ПРИЛОЖЕНИЕ Б}

\section{Четырехточечный интеграл в точке чистой модели Изинга}

Чтобы не иметь дела с расходящимися вкладами в формуле (10.14), удобно рассмотреть более общее параметрическое семейство интегралов

$$
I(\nu)=\int \frac{\left(1-x+x^{2}\right)\left(1-\bar{x}+\bar{x}^{2}\right)}{x \bar{x}(1-x)(1-\bar{x})} \frac{K(x) K(1-\bar{x})+K(\bar{x}) K(1-x)}{[x \bar{x}(1-x)(1-\bar{x})]^{\nu}} d^{2} x .
$$

Хотя эти интегралы всегда оказываются расходящимися, их можно формально вычислить при любом нецелом $\nu$, когда они стандартным образом могут быть приведены к контурным интегралам:

$$
I(\nu)=\frac{1}{2 i} \int_{0}^{1} \frac{\left(1-x+x^{2}\right) K(x)}{x^{1+\nu}(1-x)^{1+\nu}} d x \int_{C} \frac{\left(1-y+y^{2}\right) K(y)}{y^{1+\nu}(1-y)^{1+\nu}} d y,
$$

где $C$ проходит от $-\infty$ до $-\infty$, обходя точку 0 против часовой стрелки. Применяя формулы интегрирования

$$
\begin{aligned}
\int_{0}^{1} \frac{\left(1-x+x^{2}\right) K(x)}{x^{1+\nu}(1-x)^{1+\nu}} d x & =\frac{(1-2 \nu)(1-6 \nu)}{(1-4 \nu)^{2}} \frac{2^{2 \nu} \pi^{2} \Gamma^{2}(-\nu)}{\Gamma^{2}(3 / 4) \Gamma^{2}(1 / 4-\nu)} \\
\int_{0}^{1} \frac{\left(1-t+t^{2}\right) K(t)}{t^{1+\nu}(1-t)^{3 / 2-2 \nu}} d t & =\frac{(1-2 \nu)(1-6 \nu)}{(1-4 \nu)^{2}} \frac{2^{2 \nu-1} \pi \gamma(-\nu) \Gamma^{2}(3 / 4+\nu)}{\Gamma^{2}(3 / 4)}
\end{aligned}
$$

получим следующее компактное выражение:

$$
I(\nu)=\frac{2^{4 \nu-9} \pi^{2}(1-2 \nu)^{2}(1-6 \nu)^{2} \gamma^{2}(\nu-1 / 4)}{\gamma^{2}(1+\nu) \gamma^{2}(3 / 4)} .
$$

Среди многих прочих интересных свойств интеграла (Б.1) отметим, что он имеет двойной нуль в точке $\nu=1 / 6$, что подтверждает равенство нулю выражения (10.14).

Благодарности. Авторы благодарны Х. Каваи за дискуссию и интерес к работе. Ю. Ишимото благодарен И. Костову и SPTh/CEA Saclay за помощь и гостеприимство, а также М. Штудахеру и коллективу Института Альберта Эйнштейна за ценные обсуждения и гостеприимство во время посещения Потсдама. Как всегда, звезда по имени Галченок освещала путь Ал. Замолодчикова. Эта работа возникла благодаря дискуссиям, которые авторы вели во время посещения Ал. Замолодчиковым Лаборатории Теоретической Физики в RIKEN. В связи с этим он отмечает гостеприимство и стимулирующую атмосферу научного творчества в этой теоретической группе. Его работа была также поддержана Европейской Комиссией (контракт EUCLID HRPN-CT-2002-00325). 


\section{Список литературы}

[1] Al. Zamolodchikov, "Perturbed conformal field theory on fluctuating sphere. Contribution to the Balkan Workshop BW2003", Mathematical, Theoretical and Phenomenological Challenges Beyond Standard Model (29 August-02 September 2003, Vrnjancka Banja, Serbia); hep-th/0508044.

[2] V.A. Kazakov, Phys. Lett. B, 150 (1985), 282.

[3] F. David, Nucl. Phys. B, 257 (1985), 543.

[4] V. Kazakov, I. Kostov, A. Migdal, Phys. Lett. B, 157 (1985), 295.

[5] D. Boulatov, V. Kazakov, I. Kostov, A. Migdal, Nucl. Phys. B, 275 (1986), 641.

[6] A. Polyakov, Phys. Lett. B, 103 (1981), 207.

[7] T. Regge, Phys. Rev., 108 (1961), 558.

[8] V. Knizhnik, A. Polyakov, A. Zamolodchikov, Mod. Phys. Lett. A, 3 (1988), 819.

[9] F. David, Mod. Phys. Lett. A, 3 (1988), 1651; J. Distler, H. Kawai, Nucl. Phys. B, 231 (1989), 509.

[10] M. Goulian, M. Li, Phys. Rev. Lett., 66 (1991), 2051; V.S. Dotsenko, Mod. Phys. Lett. A, 6 (1991), 3601; "Correlation functions of local operators in 2-D gravity coupled to minimal matter", New Symmetry Principles in Quantum Field Theory, Proc. Adv. Study Inst. (Cargese, France), 1991. NATO Sci. Ser. B, 295, eds. J. Fröhlich, G. 't Hooft, A. Joffe et al., Plenum, NY, 1992, 432; hep-th/9110030.

[11] H. Dorn, H.-J. Otto, Phys. Lett. B, 291 (1992), 39; hep-th/9206053; Nucl. Phys. B, 429 (1994), 375; hep-th/9403141.

[12] A. Zamolodchikov, Al. Zamolodchikov, Nucl. Phys. B, 477 (1996), 577.

[13] J. Teschner, Phys. Lett. B, 363 (1995), 65; hep-th/9507109.

[14] V. Fateev, A. Zamolodchikov, Al. Zamolodchikov, Boundary Liouville field theory. I: Boundary state and boundary two-point function, hep-th/0001012; A. Zamolodchikov, Al. Zamolodchikov, Liouville field theory on a pseudosphere, hep-th/0101152.

[15] E. Brézin, C. Itzykson, G. Parisi, J.-B. Zuber, Commun. Math. Phys., 59 (1978), 35.

[16] V. Kazakov, Писъма в ЖЭТФ, 44 (1986), 105.

[17] D. Boulatov, V. Kazakov, Phys. Lett. B, 186 (1987), 379.

[18] P. Di Francesco, D. Kutasov, Phys. Lett. B, 261 (1991), 385.

[19] Y. Ishimoto, S. Yamaguchi, Phys. Lett. B, 607 (2005), 172; hep-th/0406262; Ал. Замолодчиков, ТMФ, 142:2 (2005), 218; hep-th/0505063; А. А. Белавин, Ал. Б. Замолодчиков, ТМФ, 147:3 (2006), 339 .

[20] Al. Zamolodchikov, JHEP, 0207 (2002), 029; hep-th/0109078.

[21] F. David, Nucl. Phys. B, 257 (1985), 543.

[22] D. Boulatov, V. Kazakov, I. Kostov, A. Migdal, Bulg. J. Phys., 13 (1986), 313.

[23] P. Ginsparg, G. Moore, "Lectures on 2-D gravity and 2-D string theory", TASI summer school (June 11-19, Boulder CO, 1992, USA); hep-th/9304011.

[24] Z. Burda, J. Jurkiewicz, The Ising model on a random lattice with a coordination number equal 3, Kraków University Preprint TPJU-1/89, 1989.

[25] Al. Zamolodchikov, Commun. Math. Phys., 96 (1984), 419; Ал. Б. Замолодчиков, ТМФ, 73:1 (1987), 103.

[26] Al. Zamolodchikov, Gravitational Yang-Lee model. Four point function. In preparation.

Поступила в редакцию 27.Х.2005 г. 